\title{
Coverage and Rate Analysis for Massive MIMO-enabled Heterogeneous Networks with Millimeter wave Small Cells
}

\author{
Anum Umer*, Syed Ali Hassan*, Haris Pervaiz ${ }^{\dagger}$, Qiang $\mathrm{Ni}^{\dagger}$ and Leila Musavian ${ }^{\ddagger}$ \\ *School of Electrical Engineering \& Computer Science (SEECS), National University of Sciences \& Technology \\ (NUST), Islamabad, Pakistan 44000, \{aumer.msee15seecs, ali.hassan\} @ seecs.edu.pk \\ ${ }^{\dagger}$ School of Computing \& Communications, Lancaster University, UK \{h.pervaiz, q.ni\}@ lancaster.ac.uk

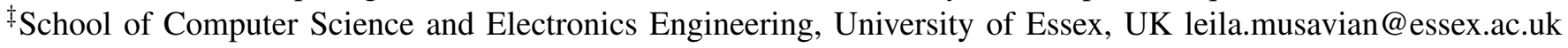

\begin{abstract}
The existing cellular networks are being modified under the umbrella of fifth generation (5G) networks to provide high data rates with optimum coverage. Current cellular systems operating in ultra high frequency (UHF) bands suffer from severe bandwidth congestion hence $5 \mathrm{G}$ enabling technologies such as millimeter wave (mmWave) networks focus on significantly higher data rates. In this paper, we explore the impact of coexistance of massive Multiple-Input Multiple-Output (MIMO) that provides large array gains and mmWave small cells on coverage. We investigate the downlink performance in terms of coverage and rate of a three tier network where a massive MIMO macro base stations (MBSs) are overlaid with small cells operating at sub-6GHz and mmWave frequency bands. Based on the existing stochastic models, we investigate user association, coverage probability and data rate of the network. Numerical results clearly show that massive MIMO enabled MBSs alongside mmWave small cells enhance the performance of heterogeneous networks (HetNets) significantly.
\end{abstract}

Index Terms-Heterogeneous networks, $5 \mathrm{G}$ cellular networks, Millimeter wave, sub-6GHz bands, massive MIMO, stochastic geometry.

\section{INTRODUCTION}

Over the years, the data rate demands have increased immensely and the exponential growth in traffic calls for making use of higher frequency bands in addition to conventional sub- $6 \mathrm{GHz}$ bands. The upcoming $5 \mathrm{G}$ technology is thought of, by researchers, as a mixture of multiple network tiers of variable sizes, transmit powers, range, operating frequencies, making a heterogeneous network (HetNet). 5G promises wide range of applications alongside high data rates but existing cellular infrastructure works on sub- $6 \mathrm{GHz}$ bands, which is unable to meet the $5 \mathrm{G}$ data demands. However, millimeter wave (mmWave) communication base stations (BSs) operating at 10 to $300 \mathrm{GHz}$ frequency bands with bandwidths up to 2 $\mathrm{GHz}$ and multiple antenna arrays at sub- $6 \mathrm{GHz}$ BSs providing high array gains are seen as key enablers of $5 \mathrm{G}$ communication providing high coverage and data rates.

The HetNets, massive Multiple-Input Multiple-Output (MIMO) and mmWave cells have gathered researchers attention and a lot of work is being done on their performance. For instance, [1] proposed stochastic geometry-based model for mmWave network to analyse coverage and rate trends, effects of antenna pattern and blockages by deploying BSs using a Poisson point process (PPP) model. Authors in [2] analysed performance of mmWave hybrid networks based on simulation models. The massive MIMO has been investigated in [3], it presents results for achievable rate in uplink network scenario for MIMO at BSs, the authors investigated user association for massive MIMO HetNets in [4] and [5] stochastically modelled user association and coverage for K-tier HetNet with massive MIMO in macro tier.

Though the aforementioned literature clearly explains the efficiency of mmWave networks in providing better coverage than UHF networks and that of massive MIMO in providing large array gains, an analytical approach to investigating the impact of co-existence of massive MIMO and mmWave cells on user association, coverage and rate in a HetNet has not been explored yet. This paper deals with extending existing stochastic geometry models for analysis of 3-tier network composed of massive MIMO enabled sub 6-GHz macro cells overlaid with mmWave and sub 6-GHz small cells. We analyse network performance in terms of user association, network coverage and data rate. To the best of our knowledge, no prior work has investigated network performance of mmWave small cells coexisting with traditional heterogeneous networks while assuming Nakagami fading model for mmWave communication and massive MIMO enabled macro BSs (MBSs). We deploy N antennas at each macro cell BS which simultaneously transmit data streams to $\mathrm{S}$ users using linear zero-forcing beamforming. The mmWave and sub-6GHz small cells BS are deployed at higher density than MBSs and user association, coverage and rate trends are discussed.

\section{SySTEM MODEL}

We consider the downlink transmission scenario of a threetier HetNet comprising of sub-6GHz macro cells overlaid with small cells operating at sub- $6 \mathrm{GHz}$ and mmWave frequency band. The BSs of $k^{\text {th }}$ tier are uniformly distributed as Homogeneous Poisson Point Process (HPPP) $\Phi_{k}$ with density $\lambda_{k}$ where $k=\{1,2,3\}$. The users are also assumed to be uniformly distributed as HPPP $\Phi_{u}$ with density $\lambda_{u}$. The sub$6 \mathrm{GHz}$ small cells constitute tier 2 while tier 3 constitutes 
small cells operating at mmWave frequency band. Massive MIMO is implemented at the macro cells where $N$ antennas are installed at each MBS which simultaneously transmit to $S$ users such that $N \gg S \geq 1$ [6]. The sub-6GHz small cell BSs, mmWave BSs and users are single antenna nodes. Zero forcing beamforming is used by each MBS for transmitting $S$ data streams with equal power assignment. Transmission is taken to be time-division duplex (TDD) and it is assumed that downlink channel state information is known at the MBS [5]. Analysis is performed for a typical user located at the origin, in accordance with Slivnyak's Theorem. The mmWave small cells can have either line of sight (LoS) or Non-line of sight (NLoS) link to the typical user. Hence we split $\Phi_{3}$, by applying independent thinning theorem, to $\Phi_{3}^{L}$ and $\Phi_{3}^{N}$ as point processes of LoS and NLoS mmWave small cells, using LoS probability function $p(R)$, to evaluate that a link of length $R$ is LoS or NLoS [1]. Thus, $\Phi_{3}^{L}$ and $\Phi_{3}^{N}$ have the densities $p(R) \lambda_{3}$ and $(1-p(R)) \lambda_{3}$, respectively, while $p(R)$ is discussed in Section II-C.

\section{A. Downlink User Association}

An open access scheme has been assumed such that user is allowed to connect to any tier BS. We assume user association is based on maximum average received power.

The average received power at a user associated with MBS $j\left(j \in \phi_{1}\right)$ is given as,

$$
P_{r, 1}=G_{M} \frac{P_{1}}{S} L_{j, M}(x),
$$

where $P_{1}$ is MBS's transmit power, $L_{j, M}(x)=x^{-\alpha_{1}}$ is path loss function where $\alpha_{1}$ is path loss exponent and $G_{M}=$ $N-S+1$ is the array gain for zero forcing beamforming transmission [6]. It is evident from (2) that the array gain of massive MIMO macro cell tier has a prominent impact on user cell association. The average received power in case the user is associated with small cell tier $i$ BS is,

$$
P_{r, i}=P_{i} L_{i}(x), \quad \text { where } i=\{2,3\},
$$

where $P_{i}$ is small cell BS transmit power in the $i^{\text {th }}$ tier and $L_{i}(x)=x_{i}^{-\alpha_{i}}$ is small cell path loss function with path loss exponent $\alpha_{i}$.

\section{B. Channel Model}

We assume independent and identically distributed (i.i.d) Rayleigh fading channel for sub-6GHz links and independent Nakagami fading for mmWave links. The SINR of a typical user located at a distance $x$ associated with the MBS is represented as

$$
\operatorname{SINR}_{M}^{u}=\frac{\frac{P_{1}}{S} h_{o, M} L_{o, M}(x)}{\sigma^{2}+\sum_{j \in \Phi_{1} \backslash b_{o, M}} \frac{P_{1}}{S} h_{j, M} L_{j, M}\left(x_{j}\right)+I_{S}},
$$

such that $I_{S}=\sum_{q \in \Phi_{2}} P_{q} h_{q} L_{q}\left(x_{q}\right)$ is inter cell interference from the small cell operating in sub-6GHz band except the serving $\mathrm{BS} b_{o, M}$, while $h_{q} \sim \exp (1)$ is the small scale fading gain from the interfering channel. Similarly, $x_{q}$ is distance between the typical user and small cell BS $q, h_{o, M} \sim \Gamma(N-$ $S+1,1)$ is the small scale fading gain of the typical user at the distance $x$ from the serving BS and $h_{j, M} \sim \Gamma(S, 1)[6], x_{j}$ is distance between typical user and $\operatorname{MBS} j, L_{q}\left(x_{q}\right)=x_{q}^{-\alpha_{2}}$ and $\sigma^{2}$ is the noise power.

Similarly, the SINR of a typical user located at the distance $x$ associated with the sub- $6 \mathrm{GHz}$ small cell is represented as

$$
\operatorname{SINR}_{S}^{u}=\frac{P_{2} h_{o, S} L_{o, S}(x)}{\sigma^{2}+I_{\overline{\mathrm{M}}}+\sum_{q \in \Phi_{2} \backslash b_{o, S}} P_{2} h_{q, S} L_{q, S}\left(x_{q}\right)},
$$

where $I_{\overline{\mathrm{M}}}=\sum_{j \in \Phi_{1}} \frac{P_{j}}{S} h_{j} L_{j}\left(x_{j}\right)$ is intercell interference from macro cells,$L_{q, S}\left(x_{q}\right)=x_{q}^{-\alpha_{2}}$ and $L_{o, S}(x)=x^{-\alpha_{2}}$. Here, $h_{q} \sim \exp (1)$ is the small scale fading gain from the interfering channel and $x_{q}$ is the distance of the typical user from small cell BS $q$. Similarly, $h_{o, S} \sim \exp (1)$ is the small scale fading gain of the typical user at the distance $x$ from the serving BS while $h_{j}$ is small scale fading power gain such that $h_{j} \sim$ $\Gamma(S, 1)$. Here, $x_{j}$ is distance of the user from MBS $j$.

The SINR for the typical user associated with mmWave small cell is represented as

$$
\operatorname{SINR}_{m}=\frac{P_{3} M_{r} M_{t} h_{o, m} L_{o, m}(x)}{\sigma^{2}+P_{3} \sum_{j \in L, N} \sum_{i \in \Phi_{3} \backslash b_{o, m}} G_{l} h_{i, m} L_{i, m}\left(x_{i}\right)},
$$

where $L_{o, S}(x)=x^{-\alpha_{3}}, h_{o, m}$ is small scale fading gain where different Nakagami fading parameters are taken for LoS and NLoS links, $M_{r}$ and $M_{t}$ are the main lobe gains of the transmit and receive antennas, $j \in\{L, N\}$ identifies the interfering link as either $\operatorname{LoS}(\mathrm{L})$ or $\operatorname{NLoS}(\mathrm{N})$ and $G_{l}$ is the directivity gain of interfering BSs. It is assumed that both the BSs and the users are in perfect alignment with each other so the directivity gain of the desired link signal is given by $M_{r} M_{t}$. Beam direction is assumed to be independently and uniformly distributed between $(0,2 \pi]$. Hence $G_{l}$ for $l=\{1,2,3,4\}$ is given as,

$G_{l}=\left\{\begin{array}{llll}a_{i}=M_{r} M_{t} & \text { with } & \text { prob. } & p_{i}=\left(\frac{\theta_{r}}{2 \pi} \frac{\theta_{t}}{2 \pi}\right) \\ a_{i}=M_{r} m_{t} & \text { with } & \text { prob. } & p_{i}=\left(\frac{\theta_{r}}{2 \pi}\left(1-\frac{\theta_{t}}{2 \pi}\right)\right) \\ a_{i}=m_{r} M_{t} & \text { with } & \text { prob. } & p_{i}=\left(\left(1-\frac{\theta_{r}}{2 \pi}\right) \frac{\theta_{t}}{2 \pi}\right) \\ a_{i}=m_{r} m_{t} & \text { with } & \text { prob. } & p_{i}=\left(\left(1-\frac{\theta_{r}}{2 \pi}\right)\left(1-\frac{\theta_{t}}{2 \pi}\right)\right) .\end{array}\right.$

\section{Blockage Model}

A stochastic blockage model is assumed for mmWave small cells, where blockages are modeled as a rectangle Boolean scheme, based on random shape theory. On the basis of this scheme, LoS probability function $p(R)$ is given by, $p(R)=$ $e^{-\beta R}$ where $R$ is the link distance and $\beta$ is dependent on statistics of blockages. The LoS probabilities for various links are assumed to be independent.

\section{PERformance AnAlysis}

In this section, we perform stochastic modeling of typical user association probability for each tier followed by coverage and rate analysis of proposed network scenario. 


\section{A. Association Probability per Tier}

1) Sub-6GHz Macro Cell tier: The association probability that a user is connected to MBS is given by,

$$
\begin{gathered}
A_{1}=2 \pi \lambda_{1} \int_{0}^{\infty} x \exp \left(-\pi \lambda_{2}\left(\frac{P_{2} S x^{\alpha_{1}}}{P_{1}(N-S+1)}\right)^{2 / \alpha_{2}}\right. \\
\left.-\pi \lambda_{1} x^{2}-2 \pi \lambda_{3}\left(\frac{P_{3} S}{P_{1}(N-S+1)}\right)^{2 / \alpha_{3}} L(x)\right) d x,
\end{gathered}
$$

where $L(x)$ is given by,

$$
L(x)=\int_{0}^{\triangle_{N}(x)} t p(t) d t+\int_{0}^{\triangle_{L}(x)} t(1-p(t)) d t,
$$

where $\triangle_{N}(x)=x^{\alpha_{N} / \alpha_{L}}$ and $\triangle_{L}(x)=x^{\alpha_{L} / \alpha_{N}} \cdot L(x)$ is based on the independent thinning of $\phi_{3}$ with LoS probability function $p(R)$, as described in section II. The probability density function of users distance to serving MBS, $f_{X_{1}}(x)$,is given as,

$$
\begin{array}{r}
f_{X_{1}}(x)=\frac{2 \pi \lambda_{1}}{A_{1}} \operatorname{xexp}\left(-\pi \lambda_{2}\left(\frac{P_{2} S x^{\alpha_{1}}}{P_{1}(N-S+1)}\right)^{2 / \alpha_{2}}\right. \\
\left.-\pi \lambda_{1} x^{2}-2 \pi \lambda_{3}\left(\frac{P_{3} S}{P_{1}(N-S+1)}\right)^{2 / \alpha_{3}} L(x)\right) .
\end{array}
$$

2) Sub-6 GHz Small Cell tier: The association probability that a user is connected to sub- $6 \mathrm{GHz}$ small cell $\mathrm{BS}$ is given by,

$$
\begin{array}{r}
A_{2}=2 \pi \lambda_{2} \int_{0}^{\infty} x \exp \left(-\pi \lambda_{2} x^{2}-2 \pi \lambda_{3} L(x)-\right. \\
\left.\pi \lambda_{1}\left(\frac{P_{1}(N-S+1) x^{\alpha_{2}}}{P_{2} S}\right)^{2 / \alpha_{1}}\right) d x,
\end{array}
$$

where $L(x)$ is given by (8). The probability density function of users distance to serving sub- $6 \mathrm{GHz}$ small cell BS, $f_{X_{2}}(x)$, is given as,

$$
\begin{array}{r}
f_{X_{2}}(x)=\frac{2 \pi \lambda_{2}}{A_{2}} \operatorname{xexp}\left(-\pi \lambda_{2} x^{2}-2 \pi \lambda_{3} L(x)-\right. \\
\left.\pi \lambda_{1}\left(\frac{P_{1}(N-S+1) x^{\alpha_{2}}}{P_{2} S}\right)^{2 / \alpha_{1}}\right) .
\end{array}
$$

3) mmWave Small Cell tier: The association probability that a user is connected to mmWave small cell BS is given as

$$
A_{3}=1-\sum_{k \in 1,2} A_{k} .
$$

The probability of associating with NLoS link is given by, $A_{N}=\Lambda_{N} \int_{0}^{\infty} \exp \left\{-2 \pi \lambda_{3} \int_{0}^{\Delta_{N}(x)} t p(t) d t\right\} f_{N}(x) d x$ [1]. Thus, the probability of associating with $\operatorname{LoS}$ link is $A_{L}=$ $1-A_{N}$. Here, $f_{N}(x)$ is probability density function of the distance of the typical user to the NLoS BS given by, $f_{N}(x)=$ $2 \pi \lambda_{3} x(1-p(x)) \exp \left(-2 \pi \lambda_{3} \int_{0}^{x} t(1-p(t)) d t\right) / \Lambda_{N}$ and for LoS link, $f_{L}(x)=2 \pi \lambda_{3} x p(x) \exp \left(-2 \pi \lambda_{3} \int_{0}^{x} t p(t) d t\right) / \Lambda_{L}$ [Eq. 4 of [1]], where $\Lambda_{N}=1-\exp \left\{-2 \pi \lambda_{3} \int_{0}^{\infty} t(1-p(t)) d t\right\}$ is probability of user having at least one NLoS link, likewise, $\Lambda_{L}=1-\exp \left\{-2 \pi \lambda_{3} \int_{0}^{\infty} t p(t) d t\right\}$ for $\operatorname{LoS}$ link. PDF of the distance of user to serving BS given it is associated with $\operatorname{LoS} \mathrm{BS}$ is $\hat{f}_{L}(x)=$
$\frac{\Lambda_{L} f_{L}(x)}{A_{L}} \exp \left\{-2 \pi \lambda_{3} \int_{0}^{\triangle_{L}(x)} t(1-p(t)) d t\right\}$ and for NLoS link $\hat{f}_{N}(x)=\frac{\Lambda_{N} f_{N}(x)}{A_{N}} \exp \left\{-2 \pi \lambda_{3} \int_{0}^{\triangle_{N}(x)} t p(t) d t\right\}$.

\section{B. Coverage Probability}

Coverage probability is the measure that the received SINR at a typical user is higher than a certain threshold, Mathematically

$$
\begin{aligned}
P_{C}^{k}(\Gamma) & =\operatorname{Pr}\left(\operatorname{SINR}_{k}>\Gamma\right) \\
& =\int_{0}^{\infty} \operatorname{Pr}\left(\operatorname{SINR}_{k}>\Gamma \mid X_{k}=x\right) f_{X_{k}}(x) d x \\
& =\int_{0}^{\infty} P^{k}(\Gamma, x) f_{X_{k}}(x) d x \quad \text { where } \quad k=\{1,2\} .
\end{aligned}
$$

The total SINR coverage probability, $P_{C}$, is calculated using law of total probability as

$$
P_{C}=\sum_{r=1}^{3} P_{C}^{r} A_{r}
$$

The coverage probability for a user associated with MBS is given by,

$$
\begin{array}{r}
P^{1}(\Gamma, x)=\sum_{l=0}^{N-S} \frac{\left(x^{\alpha_{1}}\right)^{l}}{(l !)(-1)^{l}} \sum \frac{l !}{\prod_{j=1}^{l} n_{j} !(j !)^{n_{j}}} \\
\times \exp \left(-\frac{\Gamma \sigma^{2} S x^{\alpha_{1}}}{P_{1}}-\Xi\left(\frac{\Gamma S x^{\alpha_{1}}}{P_{1}}\right)\right) \prod_{j=1}^{l}\left(\Psi^{(j)}\left(x^{\alpha_{1}}\right)\right)^{n_{j}},
\end{array}
$$

where $\Xi($.$) and \Psi^{(j)}($.$) is given as,$

$$
\begin{gathered}
\Xi(q)=2 \pi \lambda_{1} \sum_{z=1}^{S}\left(\begin{array}{l}
S \\
z
\end{array}\right)\left(\frac{P_{1}}{S}\right)^{z} q^{z}\left(\frac{\left(-q \frac{P_{1}}{S}\right)^{-z+\frac{2}{\alpha_{1}}}}{\alpha_{1}}\right) \\
B_{\left(-q \frac{P_{1}}{S} x^{-\alpha_{1}}\right)}\left[z-\frac{2}{\alpha_{1}}, 1-S\right]+2 \pi \lambda_{2} q P_{2} \frac{(J(x))^{\frac{2-\alpha_{2}}{\alpha_{2}}}}{\alpha_{2}-2}(15) \\
{ }_{2} F_{1}\left[\frac{\alpha_{2}-2}{\alpha_{2}}, 1 ; 2-\frac{2}{\alpha_{2}} ;-q P_{2}(J(x))^{-1}\right], \\
\Psi^{(1)}(i)=-\frac{\Gamma \sigma^{2} S x^{\alpha_{1}}}{P_{1}}-2 \pi \lambda_{1} S \Gamma \frac{x^{2-\alpha_{1}}}{\alpha_{1}-2} \\
\frac{{ }_{2} F_{1}\left[\frac{\alpha_{1}-2}{\alpha_{1}}, S+1 ; 2-\frac{2}{\alpha_{1}} ;-i \Gamma x^{-\alpha_{1}}\right]-2 \pi \lambda_{2} \frac{\Gamma S}{P_{1}}}{\left.\alpha_{2}-2\right)^{\frac{2-\alpha_{2}}{\alpha_{2}}} F_{1}\left[\frac{\alpha_{2}-2}{\alpha_{2}}, 2 ; 2-\frac{2}{\alpha_{2}} ;-\frac{i \Gamma S}{P_{1}} P_{2}(J(x))^{-1}\right],} \\
B_{\left(-\Gamma i x^{-\alpha_{1}}\right)}\left[j-\frac{2}{\alpha_{1}}, 1-S-j\right]+2 \pi \lambda_{2}(j !) \frac{(i)^{-j+\frac{2}{\alpha_{1}}}}{\alpha_{1}} \\
\left(-\frac{\Gamma S P_{2}}{P_{1}}\right)^{\frac{2}{\alpha_{1}}} B\left(-P_{2} \frac{\Gamma S i}{P_{1}}(J(x)){ }^{\frac{-\alpha_{1}}{\alpha_{2}}}\right)\left[j-\frac{2}{\alpha_{1}},-j\right],
\end{gathered}
$$

where $J(x)=\left(\frac{P_{2} S x^{\alpha_{1}}}{(N-S+1) P_{1}}\right)$. 
The coverage probability for a user associated with sub$6 \mathrm{GHz}$ small cell tier is given as,

$$
\begin{aligned}
& P^{2}(\Gamma, x)=\exp \left(-\frac{\Gamma \sigma^{2} x^{\alpha_{2}}}{P_{2}}-2 \pi \lambda_{1} \sum_{z=1}^{S}\left(\begin{array}{c}
S \\
z
\end{array}\right)\left(\frac{P_{1} \Gamma x^{\alpha_{2}}}{S P_{2}}\right)^{z}\right. \\
& \times \frac{\left(-\frac{\Gamma x^{\alpha_{2} P_{1}}}{S P_{2}}\right)^{-z+\frac{2}{\alpha_{1}}}}{\alpha_{1}} B_{\left(\frac{P_{1} \Gamma x_{2}}{S P_{2}}(H(x))^{-1}\right)}\left[z-\frac{2}{\alpha_{1}}, 1-S\right]- \\
& \left.2 \pi \lambda_{2} \Gamma x^{\alpha_{2}} \frac{x^{2-\alpha_{2}}}{\alpha_{2}-2} \times_{2} F_{1}\left[\frac{\alpha_{2}-2}{\alpha_{2}}, 1 ; 2-\frac{2}{\alpha_{2}} ;-\Gamma\right]\right),
\end{aligned}
$$

where $H(x)=\left(\frac{N-S+1}{S P_{2}} P_{1} x^{\alpha_{2}}\right)$. The proof can be found on similar lines as mentioned in [5] and is omitted here due to the space limitation.

The coverage probability for a user associated with mmWave small cell is given by,

$$
P_{C}^{3}(\Gamma)=A_{L} P_{3, L}(\Gamma)+A_{N} P_{3, N}(\Gamma),
$$

where $P_{3, L}$ and $P_{3, N}$ are given as,

$$
\begin{array}{r}
P_{3, L} \approx \sum_{j=1}^{N_{L}}(-1)^{j+1}\left(\begin{array}{c}
N_{L} \\
j
\end{array}\right) \times \\
\int_{0}^{\infty} \exp \left(\frac{-j \eta_{L} x^{\alpha_{L}} \Gamma \sigma^{2}}{M_{r} M_{t}}-C_{j}(\Gamma, x)-D_{j}(\Gamma, x)\right) \hat{f}_{L}(x) d x,
\end{array}
$$

and

$$
\begin{array}{r}
P_{3, N} \approx \sum_{j=1}^{N_{N}}(-1)^{j+1}\left(\begin{array}{c}
N_{N} \\
j
\end{array}\right) \times \\
\int_{0}^{\infty} \exp \left(\frac{-j \eta_{N} x^{\alpha_{N}} \Gamma \sigma^{2}}{M_{r} M_{t}}-E_{j}(\Gamma, x)-V_{j}(\Gamma, x)\right) \hat{f}_{N}(x) d x .
\end{array}
$$

where

$$
\begin{gathered}
C_{j}(\Gamma, x)=2 \pi \lambda_{3} \sum_{i=1}^{4} p_{i} \int_{x}^{\infty} F\left(N_{L}, \frac{j \eta_{L} \hat{a}_{i} \Gamma x^{\alpha_{L}}}{N_{L} t^{\alpha_{L}}}\right) p(t) t d t, \\
D_{j}(\Gamma, x)=2 \pi \lambda_{3} \sum_{i=1}^{4} p_{i} \int_{\triangle_{L}(x)}^{\infty} F\left(N_{N}, \frac{j \eta_{L} \hat{a}_{i} \Gamma x^{\alpha_{L}}}{N_{N} t^{\alpha_{N}}}\right) \\
(1-p(t)) t d t, \\
E_{j}(\Gamma, x)=2 \pi \lambda_{3} \sum_{i=1}^{4} p_{i} \int_{\triangle_{N}(x)}^{\infty} F\left(N_{L}, \frac{j \eta_{N} \hat{a}_{i} \Gamma x^{\alpha_{N}}}{N_{L} t^{\alpha_{L}}}\right) \\
V_{j}(\Gamma, x)=2 \pi \lambda_{3} \sum_{i=1}^{4} p_{i} \int_{x}^{\infty} F\left(N_{N}, \frac{j \eta_{N} \hat{a}_{i} \Gamma x^{\alpha_{N}}}{N_{N} t^{\alpha_{N}}}\right) \\
(1-p(t)) t d t
\end{gathered}
$$

and $F(N, x)=1-1 /(1+x)^{N}$. Here $\eta_{L}=N_{L}\left(N_{L} !\right)^{-\frac{1}{N_{L}}}$ and $\eta_{N}=N_{N}\left(N_{N} !\right)^{-\frac{1}{N_{N}}}$. Parameter $\hat{a}_{i}=a_{i} / M_{r} M_{t}, a_{i}$ and $p_{i}$ are defined in Section II. The proof can be found on similar lines as mentioned in [1] and is omitted here due to the space limitation.

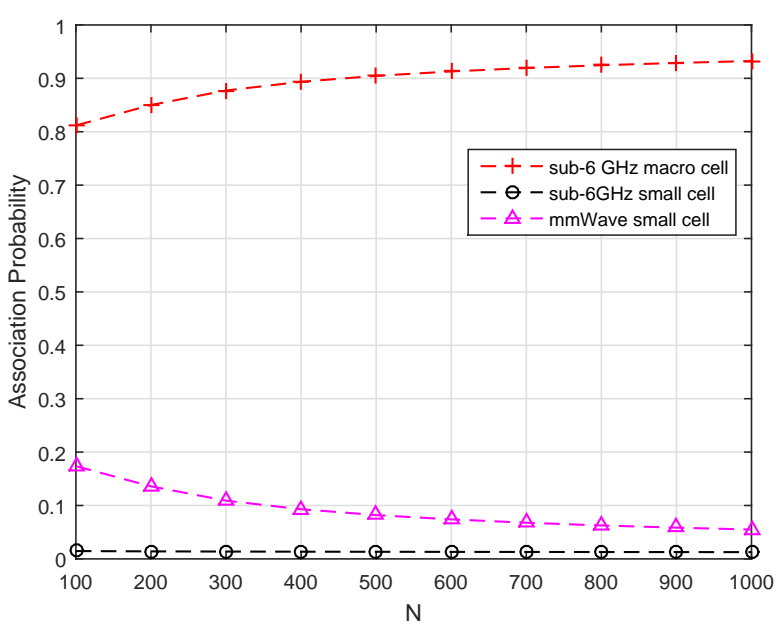

Fig. 1. Association probability verses $N$ with $\lambda_{2}=\lambda_{3}=30 \lambda_{1}$ and $S=5$

\section{Rate Coverage Probability}

We define the instantaneous downlink coverage rate for typical user such that the rate is higher than a certain threshold for an associated tier $k$ defined as

$$
\begin{aligned}
R_{C}^{k} & =\operatorname{Pr}\left(\operatorname{Rate}_{k}>\rho\right)=\operatorname{Pr}\left(W \log \left(1+\operatorname{SINR}_{k}\right)>\rho\right) \\
& =\operatorname{Pr}\left(\operatorname{SINR}_{k}>2^{\frac{\rho}{W}}-1\right)=P_{C}^{k}\left(2^{\frac{\rho}{W}}-1\right),
\end{aligned}
$$

where $W$ is the total available bandwidth at the BS.

\section{Simulation AND Numerical RESUlts}

A 3-tier HetNet is taken with MBS density $\lambda_{1}=(250000 \times$ $\pi)^{-1}, \lambda_{2}$ and $\lambda_{3}$ are taken multiples of MBS density. The sub- $6 \mathrm{GHz}$ tiers are assumed to be operating at $1 \mathrm{GHz}$ carrier frequency, $W=10 \mathrm{MHz}$, path loss exponents $\alpha_{1}=3.5$, $\alpha_{2}=4$ and transmit power $P_{1}=46 \mathrm{dBm}$ and $P_{2}=30 \mathrm{dBm}$, respectively. Likewise for mmWave tier, the operating frequency is $28 \mathrm{GHz}, W=100 \mathrm{MHz}$, path loss exponent for $\operatorname{LoS} \alpha_{L}=2$ and for $\operatorname{NLoS} \alpha_{N}=4$ and transmit power $P_{3}=30 \mathrm{dBm}$. Nakagami fading parameters for mmWave tier, $N_{N}$ and $N_{L}$, are assumed to be positive integers, 2 and 3, respectively. Array gains for all angles in main lobe are taken $M_{r}=10 \mathrm{~dB}, M_{t}=10 \mathrm{~dB}$ and for the side lobes $m_{r}=-10 \mathrm{~dB}$, $m_{t}=0 d B$. Main lobe beamwidth is taken to be $\theta_{r}=90^{\circ}$ and $\theta_{t}=30^{\circ}$. Noise $\sigma^{2}=-90 \mathrm{dBm}$ is taken with noise figure of $10 \mathrm{~dB}$. For the LoS probability function $p(R)=e^{(-\beta R)}, \beta$ is takes as $1 / \beta=141.4$ meters.

In Fig. 1 we observe the effect of increasing the number of antennas $N$ on association probability of each tier and see that association with macro BSs is directly proportional to the number of antennas on each BS and it prominently effects the user association with other tiers. This can be attributed to the higher array gains because of higher antenna density at macro BSs. Other reason is that macro BSs have greater transmit power than small cell BSs. After macro BSs most of the load is managed by mmWave BSs due to their favourable SINR distribution and larger available bandwidth compared to the sub-6GHz small cell BSs. 


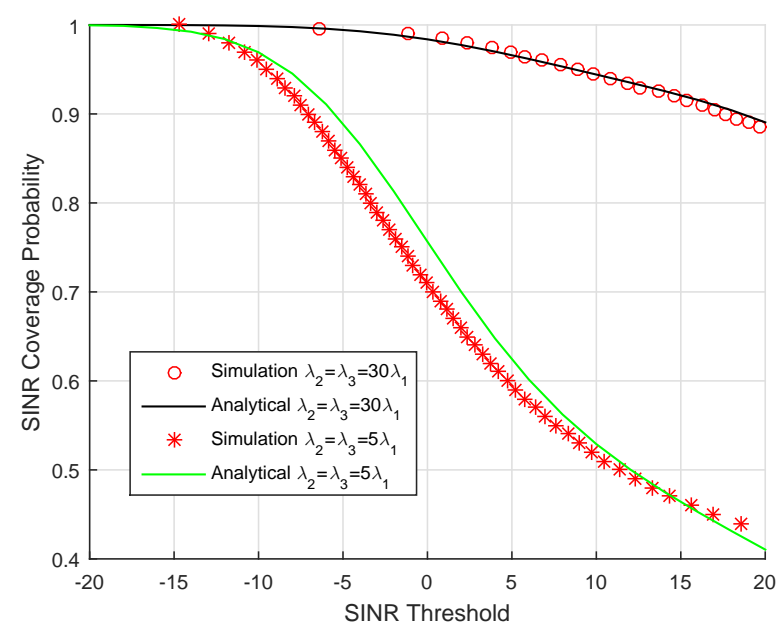

Fig. 2. SINR coverage probability $P_{C}(\Gamma)$ verses SINR threshold $\Gamma$ for $N=4$ and $S=2$

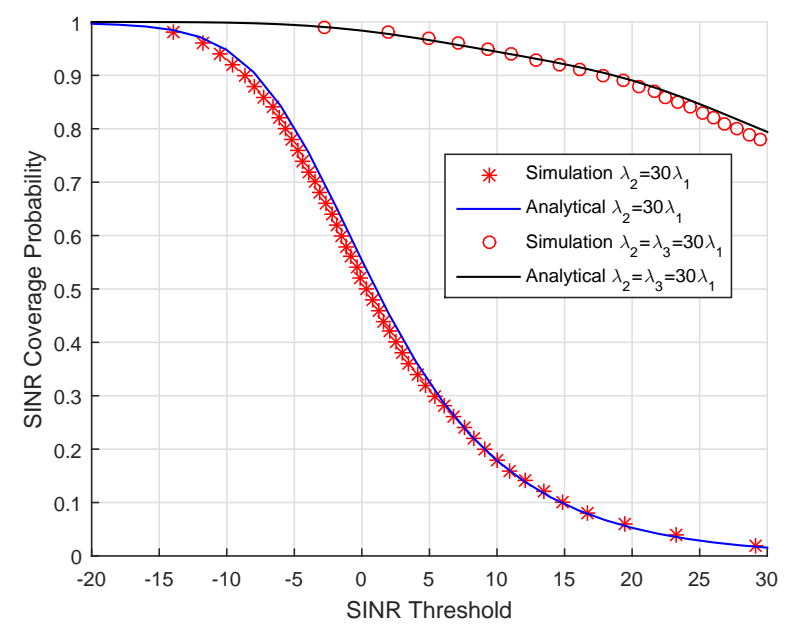

Fig. 3. SINR coverage probability $P_{C}(\Gamma)$ verses SINR threshold $\Gamma$ for $N=4$ and $S=2$

Fig. 2 shows the network coverage probability $P_{C}$ for different small cell BS densities and we observe that as small cell BS density increases, more users are offloaded to small cells and it significantly improves the SINR coverage of the network. Since small cells operate at lower power this may lead to power efficient network where high power macro BS have lower traffic but it comes at the cost of high small cell BS deployment. The macro cells may serve to provide better coverage at cell edges but within them small cells provide better coverage. Moreover, it can be observed that simulation results and analytical results are tightly bound to each other that validates the model.

Fig. 3 compares network coverage probability of 2-tier network with sub-6GHz macro and small cells and 3-tier network. We see that the mmWave tier has significant contribution to SINR coverage probability. This can be attributed to the favourable SINR distribution, larger available bandwidth and higher density of mmWave cells. Macro cells may serve to provide better coverage at cell edges but within them small

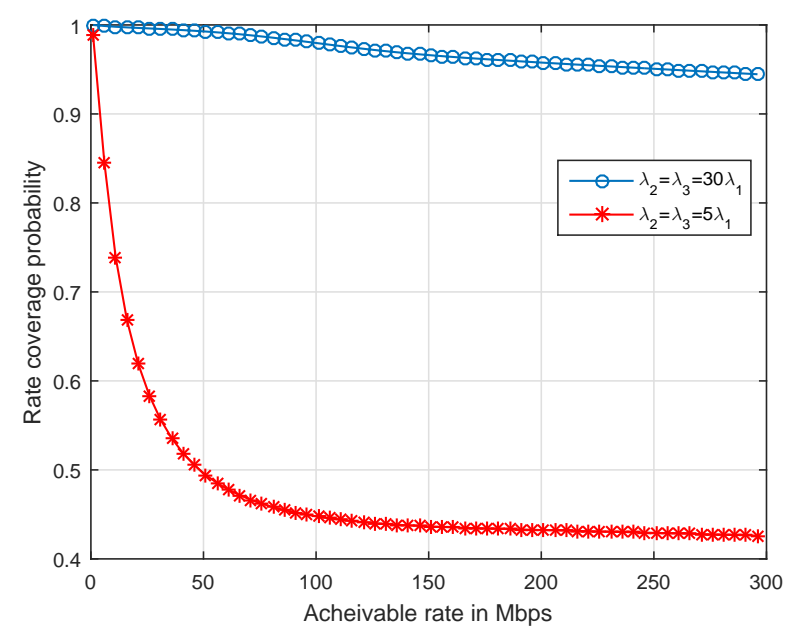

Fig. 4. Rate coverage probability $R_{C}$ verses rate threshold $\rho$ for $N=4$ and $S=2$

cells provide better coverage. Moreover, it can be observed that simulation results and analytical results are tightly bound to each other that validates the model.

In Fig. 4 we observe that as the small cell BS density increases, rate increases drastically. This is due to the fact that small cell BSs form better links to the users when their deployment density is high and users associated with mmWave small cells have greater allocated bandwidth.

\section{CONClusion}

In this paper, 3-tier HetNet coverage and rate is analysed with massive MIMO at macro tier. User association is performed based on maximum received power and effect of massive MIMO and mmWave BS density on the user cell association is investigated. It has been observed that the implementation of massive MIMO on macro tier and deployment of high density of mmWave small cells leads to significant enhancement of rate and coverage. Moreover, numerical results showed that on increasing number of antennas at macro BS, user association is biased towards macro tier, leading to low demand of small cells that simplifies the network.

\section{REFERENCES}

[1] R. W. Heath and T. Bai, "Coverage and rate analysis for millimeter-wave cellular networks, " IEEE Trans. Wireless Commun., vol. 14, no. 2, pp. 1100-1114, 2015.

[2] M. S. Omar, M. A. Anjum, S. A. Hassan, H. Pervaiz and Q. Ni, "Performance analysis of hybrid 5G cellular networks exploiting mmWave capabilities in suburban areas,"IEEE Int. Conf. Commun. (ICC), pp. 1-6, 2016.

[3] E. G. Larsson, H. Q. Ngo and T. L. Marzetta, "The multicell multiuser mimo uplink with very large antenna arrays and a finite-dimensional channel," IEEE Trans. Commun., vol. 61, no. 6, pp. 2350-2361, 2013.

[4] H. C. Papadopoulos, D. Bethanabhotla, O. Y. Bursalioglu and G. Caire, "Optimal user-cell association for massive mimo wireless networks," IEEE Trans. Wireless Commun., Vol.15, pp. 1835 - 1850, 2016.

[5] Y. Chen, M. Elkashlan, A.He, L.Wang and K-K.Wong, "Massive mimo in k-tier heterogeneous cellular networks: Coverage and rate," Proc. IEEE Global Telecommun. Conf. (GLOBECOM), pp. 1-6, 2015.

[6] W. Yu, K. Hosseini and R. S. Adve, "Large-scale mimo versus network mimo for multicell interference mitigation," IEEE J. Sel. Areas Commun, vol. 8, no. 5, pp. 930-941, Oct. 2014. 